\title{
Adaptabilidade e Estabilidade em Cultivares de Sorgo
}

\section{Jackson Silva e Oliveira1, Reinaldo de Paula Ferreira1, 2 , Cosme Damião Cruz ${ }^{3}$, Antônio Vander Pereira $^{1,4}$, Milton de Andrade Botrel ${ }^{1,5}$, Renzo Garcia von Pinho ${ }^{6}$, José Avelino Santos Rodrigues ${ }^{7}$, Fernando César Ferraz Lopes ${ }^{8}$, João Eustáquio Cabral de Miranda ${ }^{9}$}

\begin{abstract}
RESUMO - As informações sobre o comportamento de cultivares obtidas em apenas um local e ano podem não ser suficientes para recomendar uma cultivar, em função da ocorrência de interação genótipo x ambiente. Assim, a indicação de cultivares com base na análise de estabilidade e repetibilidade é mais consistente, pois possibilita prever o comportamento delas em diversos ambientes. Este trabalho estudou a adaptabilidade e estabilidade da produção de matéria seca de sete cultivares de sorgo destinadas à produção de silagem. Foram considerados dados de dois locais (Lavras e Coronel Pacheco, Minas Gerais) obtidos em dois anos agrícolas consecutivos (1997/8 e 1998/9), utilizando-se o delineamento de blocos casualizados com três repetições. A cultivar AG 2002 foi recomendada por apresentar maior rendimento, boa previsibilidade de seu desempenho e maior proximidade do padrão ideal, sendo considerada de máxima produção em qualquer ambiente. Também a cultivar Volumax pode ser recomendada por apresentar bom desempenho, desde que cultivada em ambientes mais favoráveis.
\end{abstract}

Palavras-chave: adaptabilidade, cultivares, sorgo

\section{Adaptability and Stability in Cultivars of Sorghum}

\begin{abstract}
Information about cultivars behavior obtained from only one local and year may not be enough for a cultivar recommendation due to the genotype $\mathrm{x}$ environment interaction occurrence. Then, cultivars indication based on stability and repeatability is more consistent because it enables previewing theirs performance under many environments. This work studied the adaptability and repeatability of dry matter production of seven sorghum cultivars to be used as silage. Data came from two sites (Coronel Pacheco and Lavras, Minas Gerais) and were obtained during two years (1997/98 and 1998/99) using a casual block with three replications design. Cultivar AG 2002 was recommended because showed the best productivity, good performance preview and was the cultivar that approached more to the ideal standard. Also, Volumax cultivar may be recommended due to its good performance when growing in favorable environments.
\end{abstract}

Key Words: adaptability, cultivars, sorghum

\section{Introdução}

O sorgo (Sorghum bicolor(L.) Moench) é uma planta forrageira bastante utilizada para ensilagem, pelo fato de apresentar boa produtividade de massa e características nutricionais que possibilitam obter fermentação adequada (Zago, 1991). Embora apresente, em média, valor nutritivo levemente inferior à planta do milho, o sorgo é mais tolerante à seca (Cummins, 1981; Lusk et al., 1984) e esse diferencial é importante para os sistemas de produção situados em regiões sujeitas à veranicos e que não dispõem de irrigação artificial.

Para ser recomendada, uma cultivar deve apresentar desempenho consistentemente superior em uma série de ambientes. Portanto, no estádio final de um programa de melhoramento, torna-se fundamental a avaliação do comportamento das cultivares obtidas em vários anos e locais.

O estudo das interações genótipos $\mathrm{x}$ ambientes é de grande interesse tanto para os agricultores quanto para as empresas de sementes, principalmente quando se consideram anos e locais. Interessa ao agricultor o material genético que exiba o mínimo de interação com os vários anos, de tal forma a minimizar os riscos da produção agrícola e garantir a sua receita. Para a empresa produtora de sementes tornam-se igualmente importante as interações manifestadas com os anos e com os locais. A existência de tais interações implica na necessidade de desenvolvimento de cultivares específicas para determinadas regiões, aumentando o custo de produção na manutenção e multiplicação

\footnotetext{
${ }_{1}^{1}$ Pesquisador da Embrapa Gado de Leite, Rua Eugênio do Nascimento, 610, Dom Bosco, Juiz de Fora, MG - 36.038-330

2jackoliv@cnpgl.embrapa.br; ${ }^{3}$ cdcruz@ufv.br; ${ }^{4}$ avanderp@cnpgl.embrapa.br; ${ }^{5}$ mbotrel@cnpgl.embrapa.br; ${ }^{6}$ renzo@ufla.br; ${ }^{7}$ avelino@cnpms.embrapa.br;

8 ferraze@dedalus.lcc.ufmg.br; ${ }^{9}$ jecabral@cnpgl.embrapa.br
} 
de maior número de cultivares recomendadas.

Este trabalho teve por objetivo avaliar a adaptabilidade e estabilidade da produção de matéria seca de sete cultivares de sorgo destinadas à produção de silagem.

\section{Material e Métodos}

A Embrapa Gado de Leite, juntamente com a Embrapa Milho e Sorgo, conduziram ensaios de avaliação de sorgo forrageiro em duas regiões de Minas Gerais durante dois anos agrícolas consecutivos (1997/98 e 1998/99). Os ensaios foram realizados no Sul de Minas (Lavras) e Zona da Mata (Coronel Pacheco), envolvendo sete cultivares (AG 2002, AG 2005, Volumax, BR 501, BR 601, BR 701 e Massa 3).

Os plantios foram feitos no mês de novembro, utilizando-se $70 \mathrm{~cm}$ de espaçamento entre linhas e 20 sementes/metro linear. Entre duas a três semanas após a emergência foi feito o desbate para que a população final ficasse em torno de 170.000 plantas/ha. As adubações de plantio usadas foram baseadas nas análises de solo de cada local e, em cobertura, utilizaram-se $280 \mathrm{~kg} / \mathrm{ha}$ de sulfato de amônia em aplicação única.

O delineamento experimental adotado foi o de blocos ao acaso, com três repetições. Cada parcela foi constituída por três linhas de sete metros de comprimento. As duas linhas laterais serviram de bordadura e a central foi usada para as avaliações. Também considerou-se como bordadura $1 \mathrm{~m}$ em cada extremidade da linha central, ficando a área útil de cada parcela com $3,5 \mathrm{~m}^{2}$.

A colheita foi feita quando a maioria das panículas se encontrava com os grãos entre os estádios leitoso e semi-duro. As avaliações dos estádios dos grãos foram realizadas nas bordaduras de cada uma das parcelas. No dia da colheita de cada tratamento, retiraram-se as bordaduras e as extremidades da área útil da parcela. As plantas de cada área útil foram cortadas com foice a uma altura de $10 \mathrm{~cm} \mathrm{e}$ pesadas em uma balança suspensa com precisão de 100 gramas. O material de cada parcela foi imediatamente picado em uma picadeira estacionária e pesado. Após a pesagem, o material foi misturado manualmente e três amostras de $300 \mathrm{~g}$ foram colhidas para determinação da percentagem de matéria seca. A estimativa de produção de matéria seca/ha foi feita multiplicando o peso fresco de cada parcela pela área da parcela experimental e pelo teor médio de matéria seca referente às três amostras de cada parcela.
Foi realizada a análise de variância conjunta da produção de matéria seca considerando o modelo fatorial, em que as observações foram expressas pelos efeitos de blocos dentro de anos e locais, cultivares (C), locais (L), anos (A), interações (CxL, CxA, LxA, CxAxL) e o erro aleatório. Todos os efeitos, exceto o bloco e o erro, foram considerados fixos. Foi quantificada a contribuição relativa de cada interação envolvendo cultivares, permitindo comparar a magnitude da interação temporal (CxA) e espacial (CxL). Em função dos resultados dos testes de significância da análise de variância, realizaram-se as comparações entre médias, por meio do teste de Tukey, em nível de $5 \%$ de probabilidade.

Para realizar estudos pormenorizados do comportamento das cultivares existem disponíveis várias metodologias de análise da estabilidade que diferem quanto aos conceitos utilizados para estes fenômenos e aos princípios estatísticos empregados. Muitas destas metodologias apresentam limitações de uso quando o número de ambientes em que os genótipos foram avaliados é relativamente pequeno. Neste estudo foram considerados quatro ambientes relativos aos dois anos e aos dois locais de avaliação (Coronel Pacheco e Lavras). Optou-se por empregar as metodologias propostas por Wricke (1965), Lin \& Binns (1988) e Eberhart \& Russel (1966), além do método tradicional, pela quantidade de informações que proporcionam.

Método tradicional: proporciona medida da estabilidade fenotípica expressa pela invariância do desempenho da cultivar quando avaliada em uma série de ambientes. O método considera mais estável a cultivar que apresentar a menor estimativa do quadrado médio de cultivar dentro de ambiente.

Método de Wricke: quantifica a contribuição relativa da cultivar para a soma de quadrado de cultivar $\mathrm{x}$ ambiente, estimada na análise de variância conjunta. Por este método identificam-se a cultivar de desempenho superior, considerando sua média geral, e aquela de comportamento mais previsível, em função das variações temporárias proporcionadas pelo ambiente.

Lin e Binns: trata-se de um método nãoparamétrico para avaliação da estabilidade e adaptabilidade de um grupos de cultivares. Neste caso, quantifica-se a estatística denominada Pig, que é proporcional à distância Euclidiana de uma determinada cultivar em relação ao ideal, considerada como aquela que produziria o valor correspondente máximo 
em cada ambiente. Segundo Carneiro (1998), a estatística Pig pode ser interpretada como medida de adaptação e de estabilidade de comportamento (MAEC), pois expressa o potencial produtivo da cultivar, sua invariância nos vários ambientes e sua capacidade de responder à melhoria do ambiente. As estatísticas Pifav e Pidesfav são obtidas a partir da decomposição do estimador Pig, nas partes devidas a ambientes favoráveis, que apresentam índices positivos e nas partes devidas a ambientes desfavoráveis, que apresentam índices negativos, respectivamente.

Eberhart e Russell: trata-se de um método para avaliação da estabilidade e adaptabilidade por meio da análise de regressão linear, em que se considera a produção de matéria seca em função do índice ambiental. A adaptabilidade é quantificada pelos coeficientes de regressão, procurando-se, entre as cultivares, aquela com coeficiente de regressão igual a 1,0, considerado de adaptabilidade geral. O desvio da regressão é a medida da estabilidade, que mede a previsibilidade da cultivar às variações do ambiente.

Considerações teóricas sobre estes métodos são apresentadas por Cruz \& Regazzi (1994) e Carneiro (1998). Todas as análises estatísticas foram feitas utilizando-se o programa computacional Genes (Cruz, 1997).

\section{Resultados e Discussão}

Na Tabela 1 pode ser verificado que a média de produção de matéria seca das cultivares de sorgo avaliadas foi de 18,05 t/ha, acima da média mais recente divulgada pelo IBGE para o Estado de Minas Gerais, que é de 14,18 t/ha.

O coeficiente de variação experimental apresentou magnitude de $15,80 \%$, podendo ser considerado compatível com aqueles verificados para caracteres relativos à produção de grãos, frutos ou matéria seca, geralmente de natureza genética complexa, influenciado pelo ambiente e sob controle poligênico. Foram detectadas diferenças significativas para a interação envolvendo cultivares $\mathrm{x}$ locais e cultivares $\mathrm{x}$ anos. $\mathrm{O}$ comportamento diferencial de cultivares ante as variações de anos e locais apresenta grande interesse no melhoramento, por influir na difusão de cultivares pela indústria sementeira e trazer riscos de adoção de determinada cultivar pelo agricultor. Verifica-se que a interação envolvendo anos foi mais pronunciada $\left(\mathrm{R}^{2}=54,07 \%\right)$ do que aquela envolvendo locais $\left(\mathrm{R}^{2}=26,99 \%\right)$, devendo, portanto, preocupar-se em
Tabela 1 - Análise de variância da produção de matéria seca de cultivares de sorgo avaliadas em Coronel Pacheco e Lavras - MG

Table 1 - DM production ANOVA of sorghum cultivars evaluated in Coronel Pacheco and Lavras - MG

\begin{tabular}{lcrcc}
\hline FV & GL & QM & F & $\mathrm{R}^{2}$ \\
SV & $L F$ & \multicolumn{1}{c}{$S M$} & \multicolumn{1}{c}{$F$} & $R^{2}$ \\
\hline (B/L)/A & 8 & 5,6869 & & \\
Cultivares (C) & 6 & 156,2972 & $19,1998^{* *}$ & \\
Cultivars & & & & \\
Anos (A) & 1 & 101,0863 & $17,7661^{* *}$ & \\
Years & & & & \\
Locais (L) & 1 & 702,4437 & $123,4556^{* *}$ & \\
Locals & & & & \\
Cx A & 6 & 43,8601 & $5,3879^{* *}$ & 54,07 \\
Cx L & 6 & 21,8975 & $2,6899^{*}$ & 26,99 \\
A x L & 1 & 24,9327 & $4,3819^{\text {ns }}$ & \\
C x A x L & 6 & 15,3650 & $1,8875^{\text {ns }}$ & 18,94 \\
Resíduo & 48 & 8,1405 & & \\
Residual & & & & \\
Média & & & & \\
Mean & 18,05 & & & \\
CV (\%) & & & & \\
CV & 15,80 & & & \\
\hline
\end{tabular}

${ }^{* *},{ }^{*}$ Significativo em níveis de 1 e $5 \%$ de probabilidade pelo teste $F$, respectivamente.

ns Não-significativo em nível de $5 \%$ de probabilidade.

*,* Significant at 1 and $5 \%$ of probability by $F$ test, respectively.

ns Not significant at $5 \%$ of probability.

identificar, entre as cultivares disponíveis, aquelas que apresentam boa produtividade, sem oferecer grandes oscilações no rendimento, de tal forma que a renda obtida com seu cultivo não seja afetada de um ano agrícola para outro.

$\mathrm{Na}$ Tabela 2 são apresentadas as médias de produção de matéria seca das cultivares de sorgo avaliadas em Coronel Pacheco e Lavras, no Estado de Minas Gerais. Nos dois anos de avaliação, observou-se elevada diferença entre as médias de produtividade nos dois locais estudados. A média de produtividade superior obtida em Lavras indica ser este local um ambiente superior para produção de sorgo, em relação a Coronel Pacheco. As cultivares AG 2002 e Volumax apresentaram a maior produção nos dois ambientes estudados, não diferindo estatisticamente entre si pelo teste de Tukey, em nível de 5\% de probabilidade. Por outro lado, as cultivares BR $601 \mathrm{e}$ BR 700 tiveram ampla variação de produtividade entre os locais e anos estudados.

No Tabela 3 são apresentados os resultados da análise de estabilidade e adaptabilidade utilizando o método tradicional e as metodologias propostas por Wricke (1965) e Lin \& Binns (1988). A metodologia 
Tabela 2 - Médias* de produção de matéria seca de cultivares de sorgo avaliadas em Coronel Pacheco e Lavras - MG Table 2 - DM production means of the sorghum cultivars evaluated in Coronel Pacheco and Lavras - MG

\begin{tabular}{|c|c|c|c|c|c|}
\hline \multirow{2}{*}{$\begin{array}{l}\text { Cultivares } \\
\text { Cultivars }\end{array}$} & \multicolumn{2}{|c|}{ 1997/1998 } & \multicolumn{2}{|c|}{ 1998/1999 } & \multirow[b]{2}{*}{$\begin{array}{c}\text { Média } \\
\text { Mean }\end{array}$} \\
\hline & $\begin{array}{l}\text { Coronel } \\
\text { Pacheco }\end{array}$ & Lavras & $\begin{array}{l}\text { Coronel } \\
\text { Pacheco }\end{array}$ & Lavras & \\
\hline AG 2002 & $16,26^{\mathrm{ABc}}$ & $22,64^{\mathrm{Ab}}$ & $22,92^{\mathrm{Ab}}$ & $29,22^{\mathrm{Aa}}$ & 22,76 \\
\hline AG 2005 & $10,61 \mathrm{Bab}$ & $14,38^{\mathrm{Bab}}$ & $9,06^{\mathrm{Cb}}$ & $16,06^{\mathrm{Ca}}$ & 12,53 \\
\hline Volumax & $11,71^{\mathrm{ABc}}$ & $23,74^{\mathrm{ABc}}$ & $19,40^{\mathrm{ABb}}$ & $28,47 \mathrm{ABa}$ & 20,83 \\
\hline BR 501 & $12,74^{\mathrm{ABb}}$ & $22,06^{\mathrm{Aa}}$ & $17,50^{\mathrm{ABab}}$ & $17,50^{\mathrm{Cab}}$ & 17,45 \\
\hline BR 601 & $14,87 \mathrm{ABc}$ & $17,69^{\mathrm{ABab}}$ & $21,87^{\mathrm{Aa}}$ & $21,86^{\mathrm{BCa}}$ & 19,07 \\
\hline BR 700 & $18,56^{\mathrm{Aab}}$ & $23,19^{\mathrm{Aa}}$ & $14,48 \mathrm{BC} b$ & $21,75^{\mathrm{BCa}}$ & 19,50 \\
\hline Massa 3 & $9,93^{\mathrm{Bb}}$ & $19,08^{\mathrm{ABa}}$ & $12,42 \mathrm{BC} b$ & $15,66^{\mathrm{Cab}}$ & 14,27 \\
\hline Média & 13,52 & 20,39 & 16,81 & 21,50 & \\
\hline
\end{tabular}

* Médias seguidas pelas mesmas letras, minúsculas na horizontal e maiúsculas na vertical, não diferem entre si pelo teste de Tukey, em nível de $5 \%$ de probabilidade.

* Means followed by the same letters, small in the horizontal and capital in the vertical, do not differ by Tukey test, at $5 \%$ of probability.

tradicional quantifica a invariância da cultivar diante das variações ambientais. Esta metodologia tem sido criticada na literatura, pois a invariância não tem sido considerado um atributo favorável das cultivares e normalmente está associado a cultivares de baixo desempenho. Produtores preferem que as cultivares sejam responsivas à tecnologia aplicada, sendo a invariância desejável apenas quando se deseja a rusticidade a ser manifestada em condições de emprego de baixa tecnologia. Neste estudo, os resultados observados estão de acordo com a literatura, pois a cultivar mais invariante foi a menos produtiva (AG 2005). As cultivares Volumax e AG 2002 foram as mais produtivas e apresentaram maior QMA/G, entretanto deve-se destacar a AG 2002, cuja medida da estabilidade pode ser considerada intermediária aos valores encontrados.
O método de Wricke (1965) quantifica a contribuição da cultivar para a interação genótipo x ambiente, sendo esta uma medida relativa por expressar de forma mais apropriada a imprevisibilidade do material genético avaliado. Constata-se que as cultivares de maior estabilidade foram, novamente, as menos produtivas, entretanto deve-se observar que a cultivar AG 2002 possui alta estabilidade (baixo valor de Wi), devendo ser recomendada pela sua menor oscilação na produção (Tabela 2, Figura 1).

A metodologia proposta por Lin \& Binns (1988) permite quantificar o quanto a cultivar está próxima do desempenho ideal, referenciado como sendo a de uma cultivar com a maior produção em todos os ambientes estudados. De maneira geral, esta metodologia confirma os resultados anteriormente descritos, indicando as cultivares AG2002 e Volumax

Tabela 3 - Médias de estabilidade e adaptabilidade de cultivares de sorgo avaliadas em Coronel Pacheco e Lavras MG

Table 3 - Stability and adaptability means of the sorghum cultivars evaluated in Coronel Pacheco and Lavras - MG

\begin{tabular}{|c|c|c|c|c|c|}
\hline \multirow{3}{*}{$\begin{array}{l}\text { Cultivares } \\
\text { Cultivars }\end{array}$} & \multicolumn{4}{|c|}{$\begin{array}{c}\text { Metodologia } \\
\text { Methodology }\end{array}$} & \\
\hline & \multirow{2}{*}{$\begin{array}{c}\text { Tradicional } \\
\text { QMA/G }\end{array}$} & \multirow{2}{*}{$\begin{array}{l}\text { Wricke } \\
\text { Wi }(\%)\end{array}$} & \multicolumn{3}{|c|}{ Lin e Binns } \\
\hline & & & Pig & Pifav & Pidesfav \\
\hline AG 2002 & 84,07 & 12,97 & 0,82 & 0,31 & 1,33 \\
\hline AG 2005 & 31,57 & 7,39 & 64,53 & 65,25 & 63,82 \\
\hline Volumax & 152,04 & 24,02 & 7,49 & 0,14 & 14,85 \\
\hline BR 501 & 43,47 & 11,32 & 25,43 & 35,03 & 15,83 \\
\hline BR 601 & 35,18 & 18,95 & 13,20 & 22,70 & 3,69 \\
\hline BR 700 & 44,74 & 18,73 & 15,91 & 14,03 & 17,80 \\
\hline Massa 3 & 47,32 & 6,62 & 48,70 & 51,42 & 46,17 \\
\hline
\end{tabular}

R. Bras. Zootec., v.31, n.2, p.883-889, 2002 (Suplemento) 

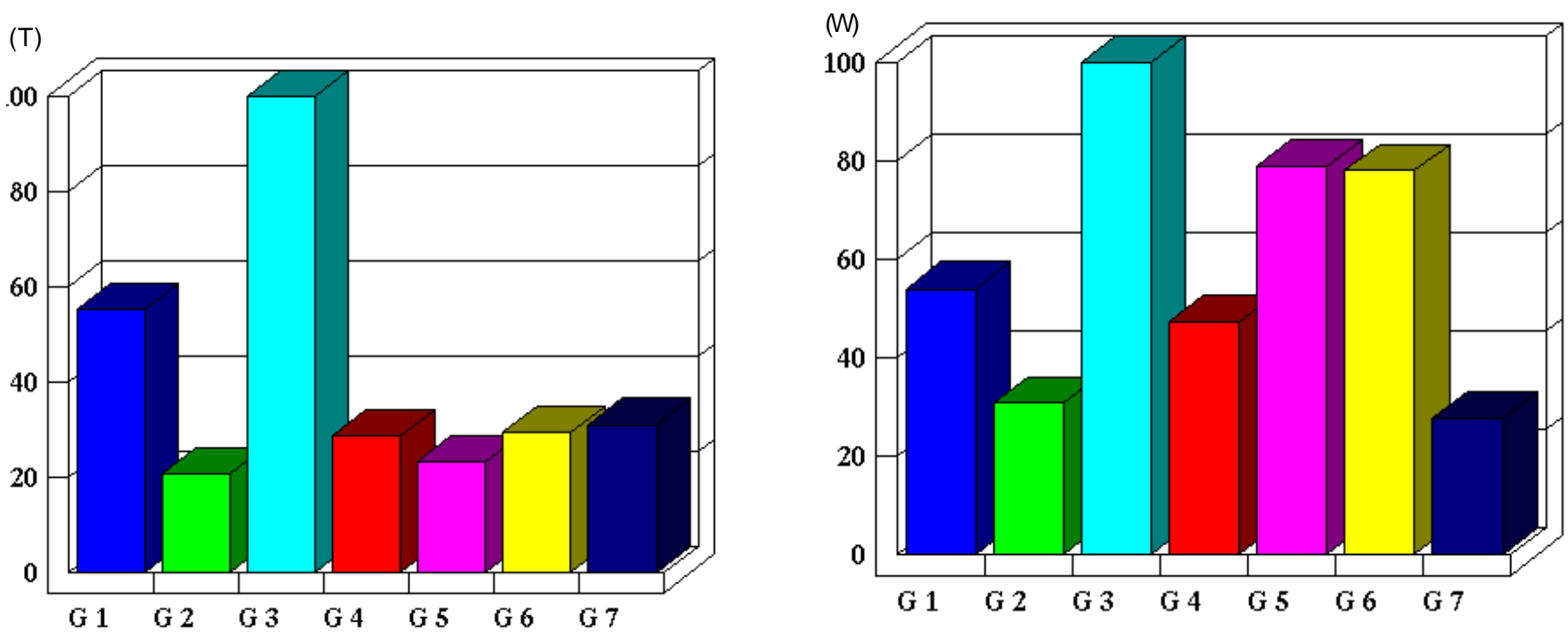

$$
\text { G1 - AG } 2002 \text { G2 - AG } 2005 \text { G3 - Volumax G4 - BR 501 G5 - BR } 601 \text { G6 - BR } 700 \text { G7 - Massa } 3
$$

Figura 1 - Medida da estabilidade de cultivares de sorgo, em relação à produção de matéria seca/ha, avaliada pelos métodos tradicional (T) e de Wricke (W).

Figure 1 - Stability of sorghum cultivars in relation to dry matter production, evaluated by traditional (T) and Wricke (W) methods.

como as mais recomendadas (Tabela 3, Figura 2). A análise nos ambientes considerados favoráveis (com produção acima da média geral) e desfavorável (com produção abaixo da média geral), permite apontar a cultivar AG 2002 como a mais apropriada, pela sua consistência em bom desempenho. Porém, verifica-se que a cultivar BR 601 poderá vir a ser uma melhor opção que a Volumax em condições de cultivo menos favorável, talvez por apresentar maior rusticidade. Esta cultivar, entretanto, não manifesta resposta à melhoria do ambiente.

A metodologia proposta por Eberhart \& Russel (1966) é, sem dúvida, a mais utilizada para inferir sobre a estabilidade e adaptabilidade de um conjunto de genótipos avaliados em uma série de ambientes. Por esta metodologia identificam-se cultivares a serem recomendadas para regiões específicas ou cultivares de ampla adaptabilidade. Utilizam-se nesta metodologia estimativas de parâmetros obtidos a partir de análise de regressão linear, como os apresentados na Tabela 4. Por esta metodologia a cultivar AG 2002 aproxima-se do ideal preconizado, pois apresenta média alta e coeficiente de regressão igual à unidade, porém mostra comportamento linear imprevisível, apesar de ter o atenuante de contar com coeficiente de determinação próximo de $80 \%$ (Figura 3).

A cultivar Volumax, também produtiva, apresenta boa previsibilidade do comportamento linear, sendo considerada recomendável para condições favoráveis, tal como verificado pela metodologia proposta

Tabela 4 - Medidas de estabilidade e adaptabilidade de cultivares de sorgo, segundo a metodologia de Eberhart e Russel

Table 4 - Stability and adaptability estimates of the sorghum cultivars evaluated by Eberhart and Russel methodology

\begin{tabular}{lcccc}
\hline $\begin{array}{l}\text { Cultivares } \\
\text { Cultivars }\end{array}$ & $\begin{array}{c}\text { Média } \\
\text { Mean }\end{array}$ & $\beta_{1 \mathrm{i}} 1$ & $\sigma_{\mathrm{di}}^{2}$ & $\mathrm{R}^{2}(\%)$ \\
\hline AG 2002 & 22,76 & $1,30 \mathrm{~ns}$ & $6,04^{*}$ & 79,17 \\
AG 2005 & 12,53 & $0,75 \mathrm{~ns}$ & $2,03 \mathrm{~ns}$ & 69,96 \\
Volumax & 20,83 & $1,93^{*} *$ & $-0,40 \mathrm{~ns}$ & 96,95 \\
BR 501 & 17,45 & $0,82 \mathrm{~ns}$ & $5,82^{*}$ & 60,74 \\
BR 601 & 19,07 & $0,56 \mathrm{~ns}$ & $8,77^{*}$ & 34,70 \\
BR 700 & 19,50 & $0,68 \mathrm{~ns}$ & $10,48^{*}$ & 41,01 \\
Massa 3 & 14,27 & $0,96 \mathrm{~ns}$ & $2,63 \mathrm{~ns}$ & 77,41 \\
\hline
\end{tabular}

1 ** Significativamente diferente de 1,0 pelo teste t, em nível de $1 \%$ de probabilidade.

2 * Significativamente diferente de zero pelo teste $\mathrm{F}$, em nível de $5 \%$ de probabilidade.

$1{ }^{* \star}$ Different significatively of 1.0 by $t$ test, at $1 \%$ of probability.

2 * Different significatively of zero by $F$ test, at $5 \%$ of probability. 

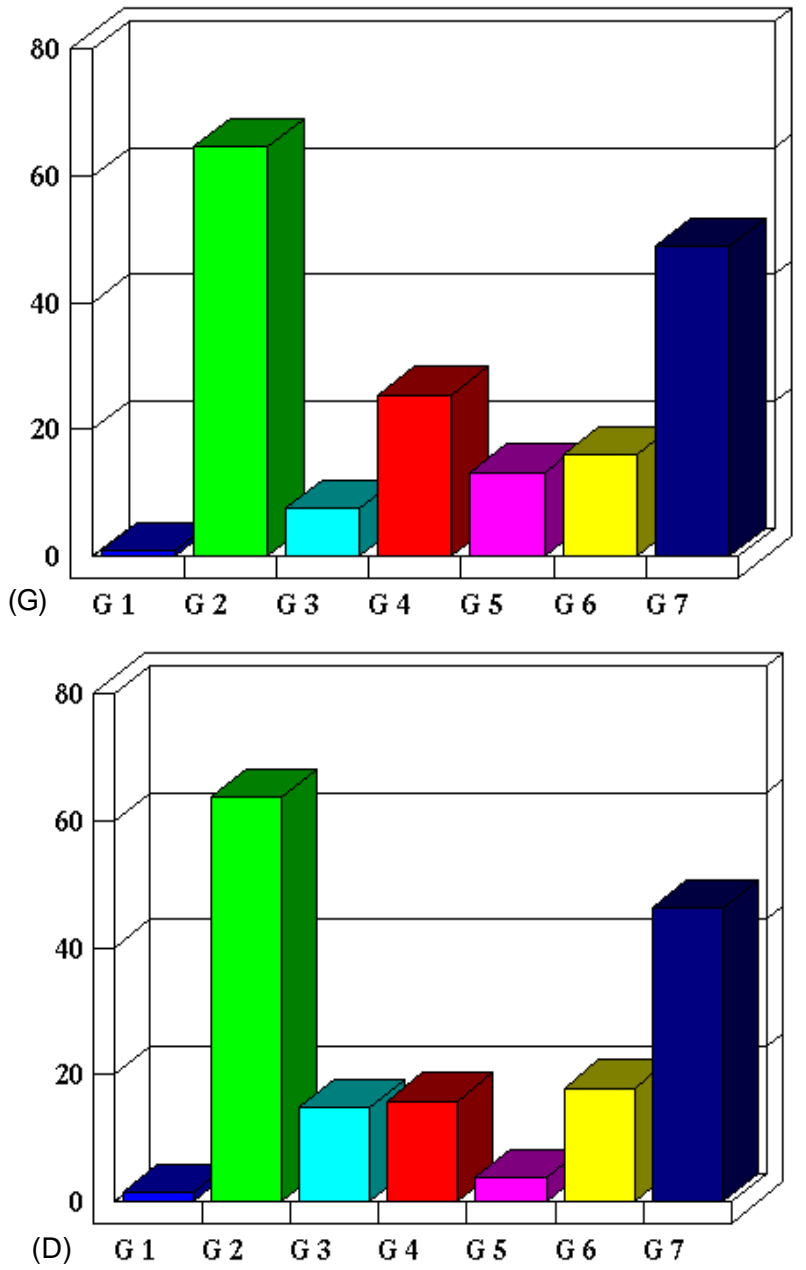

(D)

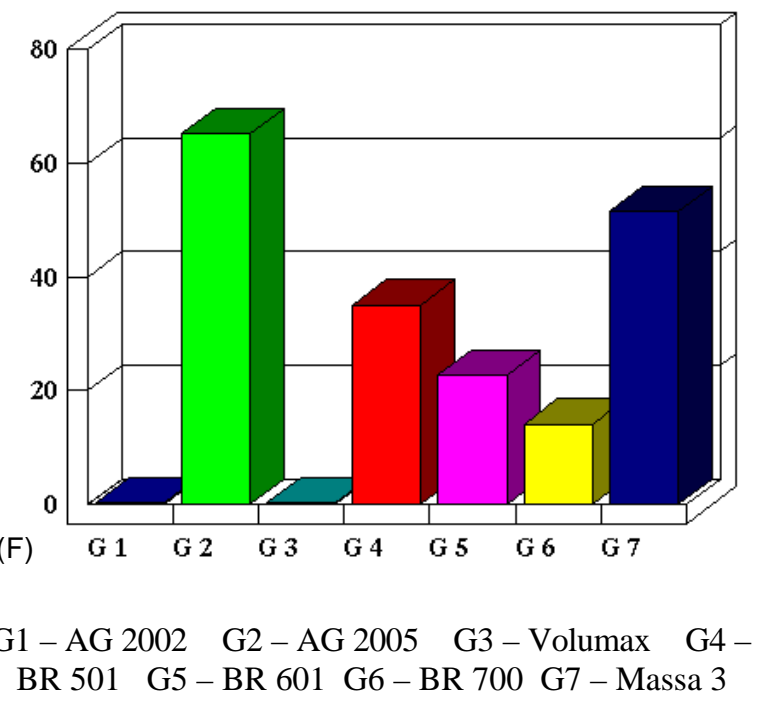

Figura 2 - Medida da estabilidade de cultivares de sorgo, em relação à produção de matéria seca/ha, avaliada pelo método de Lin e Binns, em todos os ambientes ( $G$ ), e nos ambientes desfavoráveis (D) e favoráveis (F).

Figure 2 - Stability of sorghum cultivars in relation to dry matter production (DM/ha), evaluated by Lin and Binns method, for all environments (G), and for favorables $(F)$ and unfavorables $(D)$ environments. por Lin \& Binns (1988). As cultivares BR 601 e BR 700 apresentaram coeficientes de regressão baixos, porém não estatisticamente diferentes da unidade, e apresentam baixa previsibilidade. Suas recomendações para ambientes desfavoráveis devem ser consideradas com certa cautela, exigindo maior número de avaliações para se ter conclusões mais concretas (Figura 3).

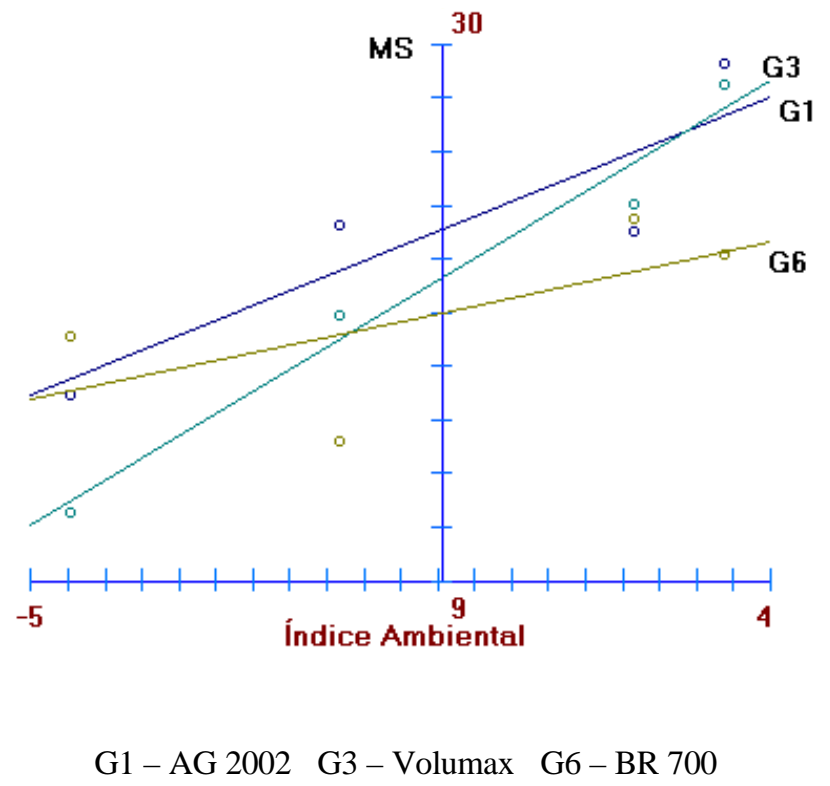

Figura 3 - Estabilidade dos cultivares de sorgo $\mathrm{G}_{1}, \mathrm{G}_{3} \mathrm{e}$ $\mathrm{G}_{6}$, em relação à produção de matéria seca/ ha, avaliada pelo método de Eberhart e Russel.

Figure 3 - Stability of $G_{1}, G_{2}$, and $G_{3}$ sorghum cultivars, in relation to dry matter production, evaluated by Eberhart and Russel method.

\section{Conclusões}

A cultivar AG 2002 pode ser recomendada pelo alto rendimento, pela boa previsibilidade e maior proximidade ao padrão ideal, considerado como o de máxima produção em todos os ambientes.

A cultivar Volumax mostrou ser outra alternativa interessante para recomendação, tendo em vista seu bom desempenho nos locais mais favoráveis. 


\section{Literatura Citada}

CARNEIRO, P.C.S. Novas metodologias de análise da adaptabilidade e estabilidade de comportamento. Viçosa, MG: Universidade Federal de Viçosa, 1998. 177p. Tese (Doutorado em Genética e Melhoramento) - Universidade Federal de Viçosa, 1989.

CRUZ, C.D. Programa GENES: aplicativo computacional em genética e estatística. Viçosa, MG: Universidade Federal de Viçosa, 1997. 442p.

CRUZ, C.D.; REGAZZI, A.J.Modelos biométricos aplicados ao melhoramento genético. Viçosa, MG: Universidade Federal de Viçosa, 1994.

CUMMINS, D.G. Yield and quality change with maturity of silage-type sorghum fodder. Agronomy Journal, v.73, p.988-990, 1981.

EBERHART, S.A.; RUSSELL, W.A. Stability parameters for comparing varieties. Crop Science, v.6, p.36-40, 1966.

LIN, C.S.; BINNS, M.R. A superiority measure of cultivar performance for cultivar $\mathrm{x}$ location data.Canadian Journal of Plant Science, v.68, p.193-198, 1988.
LUSK, J.W; KARAU, P.K.; BALOGO, D.O. et al. Brown midrib sorghum or corn silage for milk production.Journal of Dairy Science, v.67, p.1739-1744, 1984.

ZAGO, C.P. Cultura do sorgo para produção de silagem de alto valor nutritivo. In: SIMPÓSIO SOBRE NUTRIÇÃO DE BOVINOS, 4., 1991, Piracicaba.Anais... Piracicaba: Escola Superior de Agricultura Luiz de Queiroz, 1991. p.169-217.

WRICKE, G. Zur berechning der okovalenz bei sommerweizen und hafer. Pflanzenzuchtung, v.52, p.127-138, 1965.

Recebido em: 09/01/01

Aceito em: 24/11/01 